\title{
Energy Efficient Spectrum Aware Channel Sensing Routing Protocol for Cognitive Radio Mobile Ad-Hoc Networks
}

\author{
S. Chinnasamy \\ M.Phil Research Scholar \\ Department of IT, School of CSE \\ Bharathiar University, Coimbatore.
}

\author{
R. Vadivel, $\mathrm{PhD}$ \\ Assistant Professor \\ Department of IT, School of CSE Bharathiar \\ University, Coimbatore.
}

\begin{abstract}
A cognitive radio ad hoc network is the thrust research area in the field of wireless communications. This paper presents the network clustering scheme, the model and algorithm for the clustered cooperative channel sensing based on reinforcement learning. It is imperative to minimize energy cost for channel sensing so as to prolong lifetime of the network. Hence an algorithm for the cooperative channel sensing based on reinforcement learning is proposed. Performance metrics such as success rate, average broadcast delay are taken into account for comparison. Simulation results portrays that the proposed EESACSRP outperforms in terms of the chosen performance metrics.
\end{abstract}

\section{Keywords}

Cognitive Radio Ad Hoc Network, Cluster Head, Quality ofServices, cognitive radio, multi-hop architectures

\section{INTRODUCTION}

Cognitive radio is a variety of wireless communication in which a transceiver can cleverly detect which communication channels are in use and which are not and right away move into vacant channels while avoid occupied ones. This optimizes the use of available Radio-Frequency range while minimize interference to other users. In its basic form, CR is a hybrid technology concerning Software Defined Radio as applied to spread spectrum communications probable functions of cognitive radio include the capability of a transceiver to resolve its geographic location, identify and authorize its user encrypt or decrypt signals, sense nearby wireless devices in operation and change output power and modulation characteristics. Cognitive radio is the enabling technology for supporting DSA: the policy that addresses the spectrum scarcity problem that is encounter in many countries. Thus CR is regarded as one of the most hopeful technologies for future wireless communications. To make radios and wireless network truly cognitive, however, is by no way a simple task, and it requires collaborative effort from different research communities, with communications theory, networking engineering, signal processing, game theory, software-hardware design, and reconfigurable transmitter and radio-frequency design. In this paper, we provide a logical overview on Cognitive Radio networking and communications by looking at the key functions of the

Physical, Medium Access Control, and networking layers involved in a Cognitive Radio design and how these layers are crossly related. In exacting, for the PHY layer, that will address signal processing technique for spectrum sensing, supportive spectrum sensing, and transceiver design for cognitive spectrum access: for the medium access control layer is analysis sensing scheduling scheme, sensing-access exchange design, spectrum-aware access MAC, and Cognitive Radio MAC protocols. In the network layer, Cognitive Radio Network tomography, spectrum-aware routing, and Quality of-Services (QoS) control will be addressed. Emerging CRNs that are actively developed by various consistency committee and spectrum-sharing economics will also be review. Finally, point out some open questions and challenges that are related to the Cognitive Radio design.

\section{LITERATURE REVIEW}

The frequency spectrum is, indisputably, the most valuable resource in wireless communications because of its limited availability. Moreover, the use of available frequency spectrum resources is very inconsistent. There are unlicensed bands which have been overcrowded with growing technology uses, such as Bluetooth, Wi-Fi, etc. In contrast, there are licensed bands which are absolutely under-utilized. A novel solution is required in order to address both the problem where the spectrum available for certain uses is congested, and the problem where the spectrum available for other uses is allocated inefficiently. As a promising solution for this unbalanced situation, cognitive radio (CR) technology has been developed in order to enable the efficient exploitation of radio spectrum resources. CR technology has the potential to solve the wireless communications problems that result from the limited available spectrum and the inefficient use of that spectrum [1].It is the cognitive capability that enables CR to sense and capture vital information regarding the temporal and spatial variations in the existing radio environment. CR has the ability to change its transmission parameters (e.g., transmit power, modulation scheme, and operating frequency) based upon observations of, and interactions with, the surrounding environment. The CR parameters are reconfigured depending upon the characteristics of the spectrum in order to cope with the changing radio environment [2]. This CR capability opens the door for dynamic spectrum access mechanisms that allow the opportunistic use of an underutilized frequency spectrum, thereby ensuring that both the optimum spectrum and the most convenient transmission parameters are selected.

A Cognitive Radio Network (CRN) consists of wireless nodes equipped with $\mathrm{CR}$ capability that gives them a unique proficiency in sensing the frequency spectrum, reconfiguring the radios and exploiting spectrum holes based on the spectral environment [3]. Such nodes represent Secondary Users (SUs), or cognitive users. In a CRN, the licensed users are called Primary Users (PUs) and have an inherent priority to operate in certain licensed frequency bands. Whenever an SU has data to transmit, it is supposed to opportunistically use the licensed spectrum that is currently unused by a PU. Therefore, the major responsibility for any SU is to ensure the opportunistic use of the available spectrum without imposing 
any kind of interference for the PU.A Cognitive Radio Ad Hoc Network (CRAHN) [5] is a special type of CRN with no centralized network entity. As a result, SUs need cooperation schemes in order to exchange network related information, such as the presence of a PU, the node configuration, and spectrum holes. This information is obtained through local observation and spectrum sensing, and can be used for reconfiguration and routing purposes. CRAHNs are also distinguished by their inherent features, including dynamic topologies, spectrum heterogeneity, multi-hop architectures, self-configuration, and energy constrained power supplies [4]. In fact, these challenges make CRAHNs a very interesting field for researchers to work in. Consequently, a considerable amount of research and development effort has been put into ensuring CRAHNs are able to support a wide range of applications with the utmost efficiency.

\section{PROPOSED WORK}

This paper presents the network clustering scheme, the model and algorithm for the clustered cooperative channel sensing based on reinforcement learning. The clustering scheme organizes the network into structure that enhances coordination of $\mathrm{CR}$ nodes, data communication and vacant channels access in cognitive radio network. Each cluster consists of a Cluster Head (CH) [6] and non-cluster head nodes referred to as cluster Member Nodes (MNs). The MNs are ordinary nodes within the cluster that sense spectrum to detect vacant channels, detect event and communicate data to their respective $\mathrm{CH}$ via intra-cluster communications. The channel sensing problem is formulated as a Markov Decision Process (MDP) for selecting optimal set of channels to be sensed and optimal channel sensing sequence. The spectrum aware clustering scheme involves three main phases namely, initialization, set-up and maintenance phases. The initialization phase deals with neighbor discovery and determination of node eligibility $E l_{i}$ to contest for clusterhead role. The node degree denotes concentration of neighboring nodes within the node's radio range that can interact and communicate their data in a single-hop manner. High node degree indicates better opportunity to form a stable cluster that allows clusterhead to communicate with many member nodes. The number of vacant channels detected $c v_{i}$ is a key component for robust cluster structure and efficient data communications in spectrum aware clustering. Large numbers of vacant channels improve stability of the cluster and enhance both inter-cluster and intra-cluster communications since data are communicated through the vacant channels detected. The residual energy $e r_{i}$ denotes node's remaining energy which is a key factor for selecting a cluster head. High residual energy suggests longer period for the clusterhead to remain active while performing additional tasks that drain more energy from the battery of the node. The distance to sink $d c_{i}$ is an important parameter that suggests energy cost forwarding the aggregated data to the sink. Long distance attracts high energy cost for inter-cluster communications while short distance consumes less energy for inter-cluster communications.

The percentage of cluster heads $\varphi$ indicates clusters configuration for the network which determines member nodes distribution in the network. If the percentage of clusterheads is too small, significant energy will be consumed for inter-cluster communications due to large number of member nodes per cluster that may transmit over long distances. Similarly, too large percentage of clusterheads leads to high energy cost for inter-cluster communications because clusterheads may transmit at maximum power. Therefore, the percentage of clusterheads needs to be carefully chosen.

The set-up phase involves election of clusterheads based on eligibility probability $P_{i}^{g}$ and establishment of clusters where MNs identify their respective cluster and associate with the $\mathrm{CH}$ in the cluster. During the clusterhead election, SUs with highest eligibility probability $P_{i}^{g}$ among the neighboring eligible SUs would be emerged as clusterheads while the others are likely to be member nodes.

The maintenance phase allows re-election of clusterhead whenever the residual energy of the incumbent $\mathrm{CH}$ depletes to a level below a threshold $\wp$ or when the $\mathrm{CH}$ disconnected from network. The eligibility probability $P_{i}^{g}$ can be expressed as

$$
P_{i}^{g}=\frac{n d_{i} c v_{i} e r_{i} \varphi}{e m_{i} n_{z} h c_{i}}
$$

Where $e m_{i}$ denotes node's energy at full charge and $n_{z}$ denotes number of licensed channels. The clustering scheme begins at time $\tau_{s t}$ with exchange of control packets for neighbors discovery and then follows by sensing predefined set of licensed channels to detect availability or otherwise of PU on the licensed channels. Each $S U_{i}$ computes its eligibility probability $P_{i}^{g}$ and then compares it with a given threshold $\Omega_{e}$ to determine its eligibility $E l_{i}$ to contest for the clusterhead position.

A SU is said to be eligible $E l_{i}$ to contest for cluster head if its eligibility probability $\left(P_{i}^{g} \geq \Omega_{e}\right)$ is greater than or equal to a given threshold $\Omega_{e}$. This means that SUs with eligibility probability $\left(P_{i}^{g} \geq \Omega_{e}\right)$ less than a given threshold $\Omega_{e}$ are likely to remain as member node.

The cluster heads are model as teach agents which sequentially sense finite number of channels, and learn optimal set of channels by adopting a RL technique.

\subsection{Energy cost}

Energy cost for channel sensing in CR-WSN constitutes significant portion of the network energy consumption. Therefore, it is imperative to minimize energy cost for channel sensing so as to prolong lifetime of the network. Energy dissipated by $S U_{i}$ for listening to the channel $c h_{z}$, receiving $N_{0}$ signal samples for a minimum duration of $T_{c s}$ and processing the received signals for local decision can be expressed as: 


$$
E_{c s}=T_{c s} P_{e d}+E_{s p},
$$

Where $P_{e d}$ denotes energy detector's circuit power consumption and $E_{s p}$ denotes energy consumption for processing the received $N_{0}$ signals samples. Therefore, energy cost for channel sensing is heavily influenced by the sensing duration, minimum sensing time always minimizes energy cost for channel sensing but this may not guarantee accurate sensing results.

The narrow band cooperative channel sensing algorithm begins with initializing the array of all the state-action values $Q(|S|,|A|)$ to zero (line 2) and then performing iterations (line 3 to line 14) up to the number of episodes $E_{p s d}$ specified in the inputs (line 1). State transition is sequential (line 4) and restarted whenever the next state reaches the number of channels (line 12). In each state of the episodes (line 5), a softmax action selection strategy chooses an action with highest state-action value through exploration of random actions.

The corresponding channel parameters i.e. channel bandwidth, required bandwidth, $\mathrm{PU}$ birth rate, $\mathrm{PU}$ death rate as specified in the inputs are used to compute the rewards based on three metric functions namely channel sensing energy cost, channel availability and local decision accuracy (line8). The cumulative reward (line9) which is the weighted average of the three computed rewards is used to update the Q-values (line 10) as described. At the end of the episodes, a final Qmatrix consist all the state-action values would be obtained and the highest value (line15) of each row in the Q-table is determined.

An optimal policy, which is column index of each row in the Q-table that returns the maximum value, denotes the selected channel in that state and the sequence of the channels in the optimal Q-table denotes the optimal sequence of channel sensing $S_{e q}^{*}$ (line 17).The selected channels in the optimal Qtable form the optimal set of channels $A_{e x}^{*}$ (line 18 to line 21).

Algorithm for the cooperative channel sensing based on reinforcement learning

\section{Algorithm 1: RL based Cooperative channel Sensing}

1:input

$|S|,|A|,|H|,\left|B_{w}\right|,\left|r_{0}\right|,\left|r_{1}\right|, E_{p s d}, w_{\alpha_{1}}, w_{\alpha_{2}}, w_{\alpha_{3}}, r q B_{w}$

2: Initialize: $Q(|S|,|A|) \leftarrow 0, k \leftarrow 0, a_{k}^{e} \leftarrow 0$

3: for $e \leftarrow 1$ to $E_{p s d}$ do

4: $k \leftarrow k+1$

5: $\quad a_{k}^{e} \leftarrow$ action Strategy $\left(s_{k}^{e}, Q\right)$

6: $\quad$ if $a_{k}^{e} \neq 0$ and $k \leq n_{z}$ then

7: Sense Channel $\left(C h_{z}, a_{k}^{e}=z\right)$
8: Compute

$r w_{a k+1}^{e} \leftarrow \frac{1}{2} \operatorname{sum}\left(\frac{t_{i d}}{t_{b}}, \frac{r q B_{w}}{B_{w}}\right), r w_{l k+1}^{e} \leftarrow \frac{1}{2} \operatorname{sum}\left(L D_{i}^{z}, C D_{j}^{z}\right)$,

$r w_{e k+1}^{e} \leftarrow \tau_{a v} / T_{c s}$,

9: Compute

$r_{k+1}^{e} \leftarrow$ Weighted Average $\left(r w_{a k+1}^{e}, r w_{l k+1}^{e}, r w_{e k+1}^{e}\right)$

10: update $Q_{k}^{e} \leftarrow Q_{k}^{e}+\alpha\left[r_{k+1}^{e}+\gamma \max _{a}\left(Q_{k+1}^{e}\right)-Q_{k}^{e}\right]$

11: else

12: $k=0$

13: end if

14: end for

15: $\left[H_{\text {seq }}, I\right] \leftarrow \max Q$

16: $j=1, i=j+1$

17: $S_{\text {eq }}^{*}=\left|H_{\text {seq }}\right|$

18: $\quad$ while $i \leq n_{z}+1$ do

19: if $I(j) \neq 0$ and $I(j) \neq I(i)$ then

20: $A_{e x}^{*} \leftarrow|I|$

21: end if

22: end while

\section{SIMULATION SETTINGS}

Table - 1

\begin{tabular}{|l|c|}
\hline Number of SUs N & 16 \\
\hline Number of PUs K & 40 \\
\hline Number of Channels M & 20 \\
\hline $\begin{array}{l}\text { Side length of the } \\
\text { simulation area L }\end{array}$ & 10 (unit length) \\
\hline $\begin{array}{l}\text { Radius of the sensing } \\
\text { range } r_{s}\end{array}$ & 2(unit length) \\
\hline $\begin{array}{l}\text { Radius of the transmission } \\
\text { range } r_{c}\end{array}$ & 2(unit length) \\
\hline $\begin{array}{l}\text { Number of selected } \\
\text { channels } n\end{array}$ & 1 \\
\hline $\begin{array}{l}\text { The normalized PU arrival } \\
\text { rate } \lambda_{p}\end{array}$ & 10 (times slots) \\
\hline $\begin{array}{l}\text { The PU Packet length } L_{p} \\
\text { The probability of a } \\
\text { successful transmission } \sigma\end{array}$ \\
\hline
\end{tabular}


5. RESULTS AND DISCUSSIONS

5.1.Number of Secondary Users Vs Success Rate

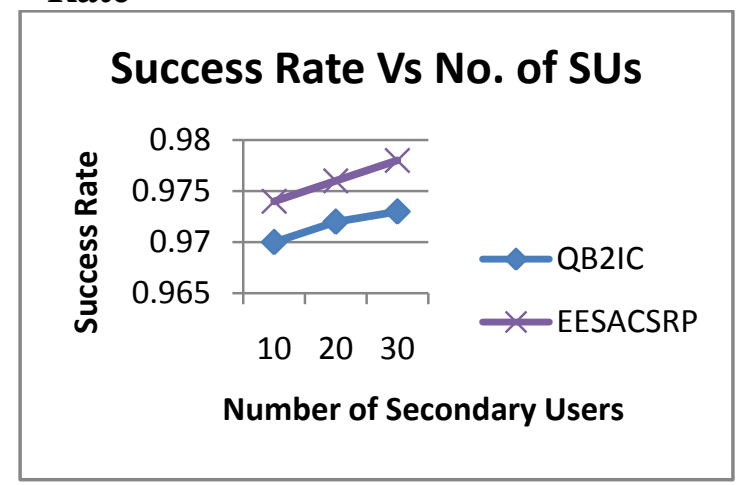

Figure.5.1. Number of Secondary Users Vs Success Rate

Table.5.1. Number of Secondary Users Vs Success Rate

\begin{tabular}{|c|c|c|}
\hline & \multicolumn{2}{|c|}{ Protocols } \\
\hline No. of SUs & QB2IC & EESACSRP \\
\hline 4 & 0.89 & 0.95 \\
\hline 9 & 0.83 & 0.88 \\
\hline 16 & 0.82 & 0.83 \\
\hline
\end{tabular}

Fig.5.1. portrays the success rate performance subject to increasing the number of secondary users of the EESACSRP compared with QB2IC. It is evident that EESACSRP attains better success rate than that of QB2IC protocols. The simulation result values are shown in Table 5.1.

\subsection{Number of Secondary Users Vs Average Broadcast Delay}

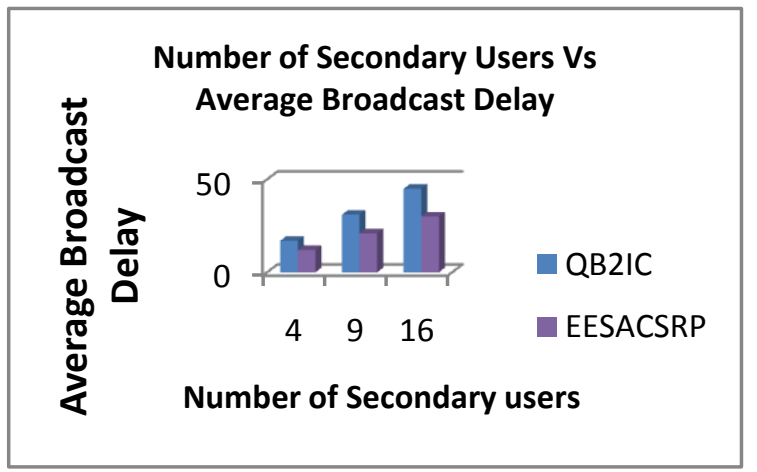

Figure.5.2. Number of Secondary Users Vs Average Broadcast Delay

Fig.5.2. Projects the average broadcast delay performance subject to increasing the number of secondary users of the EESACSRP compared with QB2IC. It is obvious that EESACSRP attains less broadcast delay than that of QB2IC protocols. The simulation result values are shown in Table 5.2 .
Table.5.2. Number of Secondary Users Vs Average Broadcast Delay

\begin{tabular}{|c|c|c|}
\hline & \multicolumn{2}{|c|}{ Protocols } \\
\hline No. of SUs & QB2IC & EESACSRP \\
\hline 4 & 17 & 12 \\
\hline 9 & 31 & 21 \\
\hline 16 & 45 & 30 \\
\hline
\end{tabular}

\subsection{Number of Primary Users Vs Success} Rate

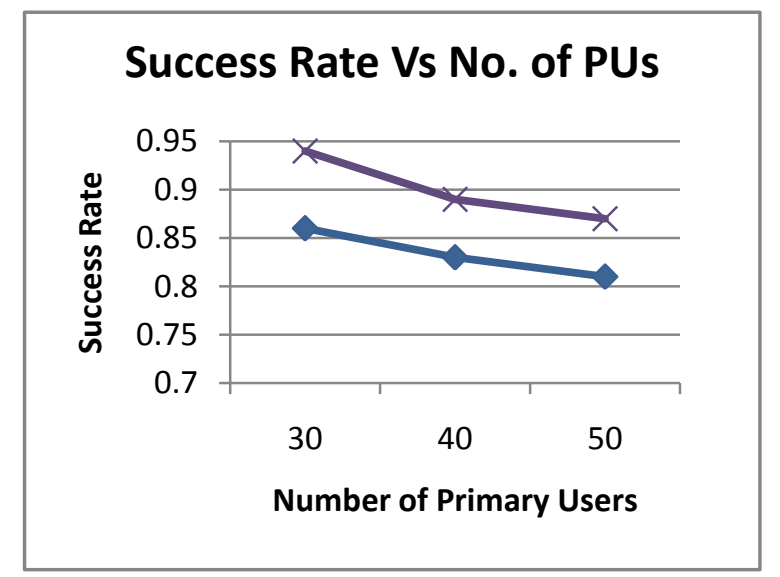

Figure.5.3. Number of Primary Users Vs Success Rate

Fig.5.3. Projects the success rate performance subject to increasing the number of primary users of the EESACSRP compared with QB2IC. It is certain that EESACSRP attains increased success rate than that of QB2IC protocols. The simulation result values are shown in Table 5.3.

Table.5.3. Number of Primary Users Vs Success Rate

\begin{tabular}{|c|c|c|}
\hline & \multicolumn{2}{|c|}{ Protocols } \\
\hline No. of SUs & QB2IC & EESACSRP \\
\hline 30 & 0.86 & 0.94 \\
\hline 40 & 0.83 & 0.89 \\
\hline 50 & 0.81 & 0.87 \\
\hline
\end{tabular}

\subsection{Number of Primary Users Vs Average Broadcast Delay}

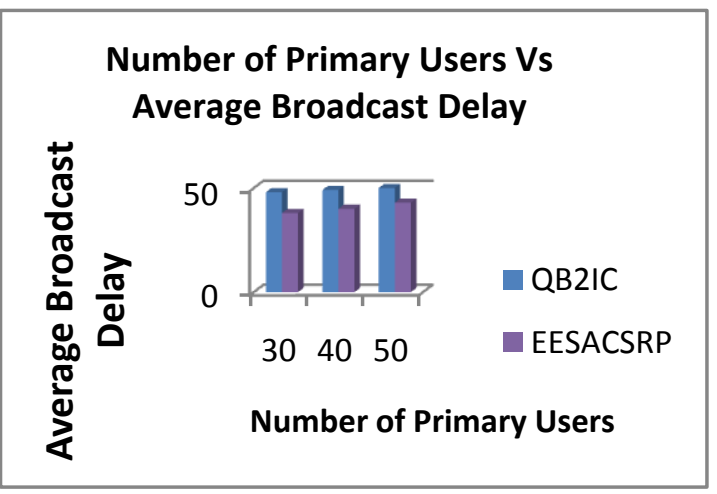

Figure 5.4 Number of Primary Users Vs Average Broadcast Delay 
Fig.5.4. showcases the average broadcast delay performance subject to increasing the number of primary users of the EESACSRP compared with QB2IC. It is evident that EESACSRP attains less broadcast delay than that of QB2IC protocols. The simulation result values are shown in Table.5.4.

Table 5.4 Number of Primary Users Vs Average Broadcast Delay

\begin{tabular}{|c|c|c|}
\hline & \multicolumn{2}{|c|}{ Protocols } \\
\hline No. of SUs & QB2IC & EESACSRP \\
\hline 30 & 48 & 38 \\
\hline 40 & 49 & 40 \\
\hline 50 & 50 & 43 \\
\hline
\end{tabular}

\subsection{Number of Channels Vs Success Rate}

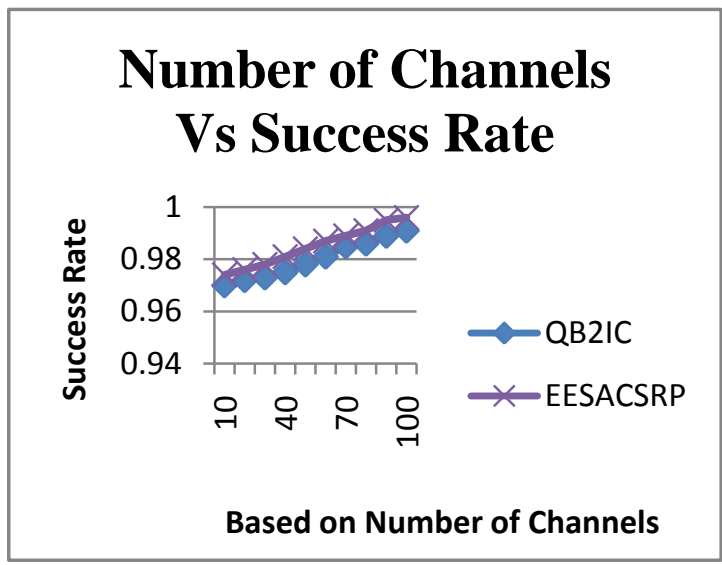

Figure 5.5 Number of Channels Vs Success Rate

Fig.5.5. envisage the success rate performance subject to increasing the number of channels of the EESACSRP compared with QB2IC. It is certain that EESACSRP attains increased success rate than that of QB2IC protocols. The simulation result values are shown in Table 5.5.

Table 5.5 Number of Channels Vs Success Rate

\begin{tabular}{|c|c|c|}
\hline & \multicolumn{2}{|c|}{ Protocols } \\
\hline No. of SUs & QB2IC & EESACSRP \\
\hline 10 & 0.97 & 0.974 \\
\hline 20 & 0.972 & 0.976 \\
\hline 30 & 0.973 & 0.978 \\
\hline 40 & 0.975 & 0.981 \\
\hline 50 & 0.978 & 0.984 \\
\hline 60 & 0.981 & 0.987 \\
\hline 70 & 0.985 & 0.989 \\
\hline 80 & 0.986 & 0.991 \\
\hline 90 & 0.989 & 0.995 \\
\hline 100 & 0.991 & 0.996 \\
\hline
\end{tabular}

\subsection{Number of Channels Vs Average Broadcast Delay}

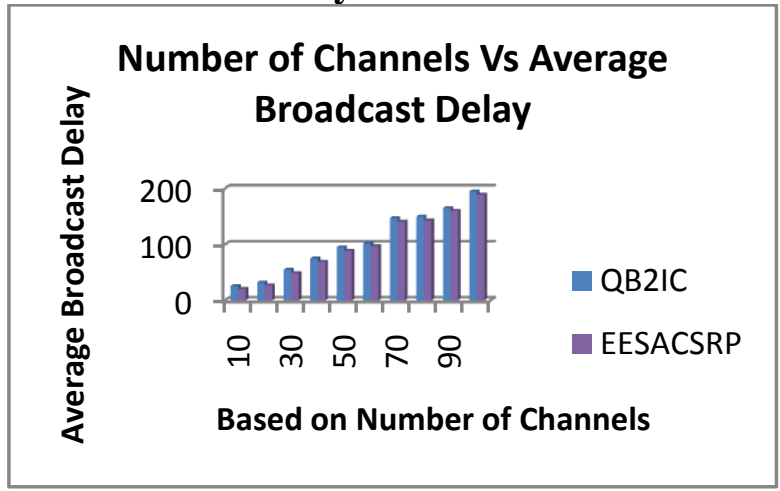

Figure 5.6 Number of Channels Vs Average Broadcast Delay

Fig.5.6. projects the average broadcast delay performance subject to increasing the number of channels to the EESACSRP compared with QB2IC. It is obvious that EESACSRP attains less broadcast delay than that of QB2IC protocols. The simulation result values are shown in Table.5.6.

Table 5.6 Number of Channels Vs Average Broadcast Delay

\begin{tabular}{|c|c|c|}
\hline & \multicolumn{2}{|c|}{ Protocols } \\
\hline No. of SUs & QB2IC & EESACSRP \\
\hline 10 & 26 & 21 \\
\hline 20 & 32 & 27 \\
\hline 30 & 55 & 49 \\
\hline 40 & 75 & 69 \\
\hline 50 & 95 & 89 \\
\hline 60 & 102 & 97 \\
\hline 70 & 147 & 141 \\
\hline 80 & 150 & 143 \\
\hline 90 & 165 & 160 \\
\hline 100 & 195 & 189 \\
\hline
\end{tabular}

5.7.Number of Unsynchronized Time Slots Vs Success Rate

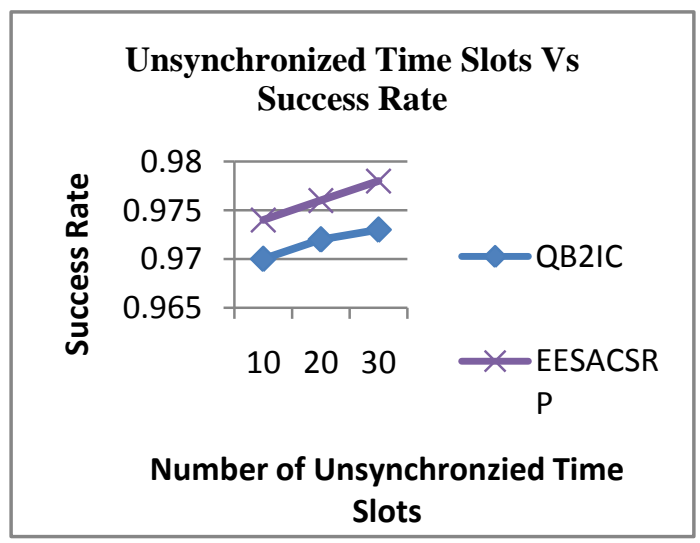

Figure 5.7 Number of Unsynchronized Time Slots Vs Success Rate 
Fig.5.7. envisages the success rate performance subject to increasing the unsynchronized time slots to the EESACSRP compared with QB2IC. It is certain that EESACSRP attains increased success rate than that of QB2IC protocols. The simulation result values are shown in Table 5.7.

Table 5.7 Number of Unsynchronized Time Slots Vs Success Rate

\begin{tabular}{|c|c|c|}
\hline & \multicolumn{2}{|c|}{ Protocols } \\
\hline No. of SUs & QB2IC & EESACSRP \\
\hline 4 & 0.93 & 0.99 \\
\hline 9 & 0.89 & 0.97 \\
\hline 16 & 0.89 & 0.95 \\
\hline
\end{tabular}

\subsection{Number of Unsynchronized Time Slots Vs Average Broadcast Delay}

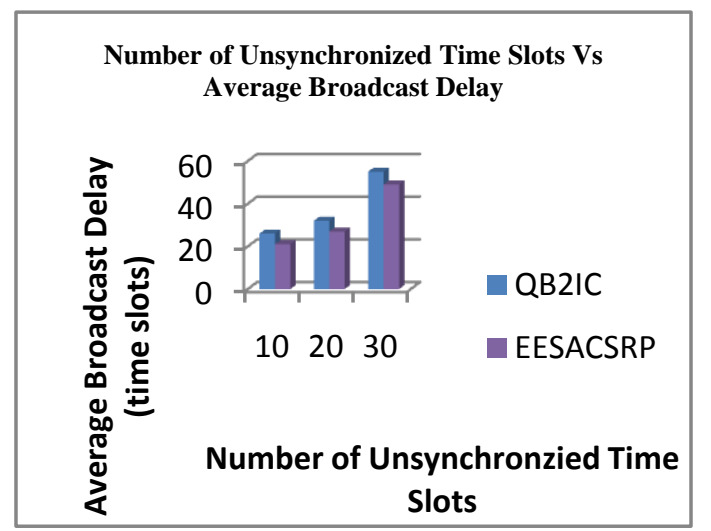

Figure 5.8 Number of Unsynchronized Time Slots Vs Average Broadcast Delay

Fig.5.8.projects the average broadcast delay performance subject to increasing the number of unsynchronized time slots to the EESACSRP compared with QB2IC. It is obvious that EESACSRP attains less broadcast delay than that of QB2IC protocols. The simulation result values are shown in Table 5.8 .

Table 5.8 Number of Unsynchronized Time Slots Vs Average Broadcast Delay

\begin{tabular}{|c|c|c|}
\hline & \multicolumn{2}{|c|}{ Protocols } \\
\hline No. of SUs & QB2IC & EESACSRP \\
\hline 4 & 20 & 15 \\
\hline
\end{tabular}

\begin{tabular}{|c|c|c|}
\hline 9 & 38 & 30 \\
\hline 16 & 51 & 43 \\
\hline
\end{tabular}

\section{CONCLUSION AND FUTURE WORK}

This paper presents the network clustering scheme, the model and algorithm for the clustered cooperative channel sensing based on reinforcement learning. It is imperative to minimize energy cost for channel sensing so as to prolong lifetime of the network. Performance metrics such as success rate, average broadcast delay are taken into account for comparison. Simulation results portrays that the proposed EESACSRP outperforms in terms of the chosen performance metrics.

\section{REFERENCES}

[1] QB2IC: A QoS-Based Broadcast Protocol Under Blind Information for Multihop Cognitive Radio Ad Hoc Networks Yi Song, Member, , and Jiang Xie, Senior Member, IEEE transactions on vehicular Technology, vol. 63 , no. 3 , march 2014

[2] kyildiz IF, Lee WY, Chowdhury KR. Crahns: Cognitive radio ad hoc networks. AD hoc networks 2009a 7(5):810-36.

[3] Akyildiz IF, Lee WY, Vuran MC, Mohanty S. Next generation/dynamic spectrum access/cognitive radio wireless networks: a survey. Computer networks 2006; 50(13):2127-59.

[4] Cesana M, Cuomo F, Ekici E. Routing in cognitive radio networks: Challenges and solutions. Ad Hoc Networks 2011; 9(3):228-48

[5] Rahman M, et al. Cognitive Radio Ad-hoc Networks: A Routing Perspective, 2013.

[6] Yang Qina,*, Xiaoxiong Zhonga,b, Yuanyuan Yangc, Li Lia, Fangshan Wua,Y. Qin et al., TCPJGNC: A transport control protocol based on network coding for multi-hop cognitive radio networks, Computer Communications (2016).

[7] On-demand routing protocols for cognitive radio ad hoc networks, Shelly Salim and Sangman Moh, EURASIP Journal on Wireless Communications and Networking (2013). 